\title{
Vaccination for Covid-19 and Human Papillomavirus: A Comparative Study
}

\section{Jitendar Sharma ${ }^{1}$, Umesh Gupta ${ }^{2}$, Pudi Nagaseshu ${ }^{3 *}$, Kavita Kachroo ${ }^{4}$ and Greeshma Gopalan ${ }^{5}$}

${ }^{1}$ MD \& CEO/ED, Kalam Institute of Health Technology, Andhra Pradesh Med Tech Zone, Visakhapatnam, India

${ }^{2}$ Professor, Head of Mats School of Business Studies, MATS Institute of Management and Entrepreneurship, Bangalore, India

${ }^{3}$ Scientist- C, Kalam Institute of Health Technology, Andhra Pradesh Med Tech Zone, Visakhapatnam, India

${ }^{4}$ Scientist- D, Kalam Institute of Health Technology, Andhra Pradesh Med Tech

Zone, Visakhapatnam, India

${ }^{5}$ Scientist- A, Kalam Institute of Health Technology, Andhra Pradesh Med Tech

Zone, Visakhapatnam, India

*Corresponding Author: Pudi Nagaseshu, Scientist- C, Kalam Institute of Health Technology, Andhra Pradesh Med Tech Zone, Visakhapatnam, India.
Received: November 25, 2021

Published: December 29, 2021

(c) All rights are reserved by Pudi Nagaseshu., et al.

\begin{abstract}
Introduction: This review focused on two scenarios like HPV vaccination and Covid-19 vaccination. In the First scenario Covid 19 Vaccinations are known to boost immune system and it acts as a shield which fight against virus. In India Bharat Biotech in collaboration with the Indian Council of Medical Research had developed a vaccine Covaxin. The importance of covid-19 vaccination is to avoid severity and deaths from corona virus. Although, HPV vaccine and Covid 19 vaccines are different from each other and they are different in formulations affect and their usage, storage and type of administration, they are to be safe and effective. Hence, this article focusses on both covid 19 and HPV vaccination showing difference in costing, lives saved and budgetary impact. In the second scenario the importance of HPV vaccine is it protects against certain types of HPV that can lead to genital warts or cancer. In 2019 National immunization programs have been implemented in 124 countries and thus most of the girls and women were vaccinated by preventing cervical cancer.

Results and Discussion: Vaccines are saving millions of lives each year and they are most reliable and cost-effective for public health interventions. The efficacy of different Covid -19 vaccination m-RNA-1273 (70.4\%), AstraZeneca (78\%), Sinovac (94.5), Sputnik (91.4), Covaxin (81\%), Covishiled (90\%), m-RNA-BNT162B2 (94.1\%). Gardasil has 98\% efficacy and the efficacy of Cervarix is $91 \%$. Conclusion: The efficacy of Covid 19 vaccination saves lives and 2,07,421 lives saved correspond to 1,506,501 QALYs gained for covid 19. Total Lives saved by HPV vaccination for 9 to 14 -year-old is lesser than 42,000 annually.
\end{abstract}

\section{Abbreviations}

WHO: World health Organization; HPV: Human Papillomavirus; RCTS: Randomized Control Trails

\section{Introduction}

Coronavirus pandemic, was seen in Wuhan city of China in 2019 and which spreads all around the world.

SARS-CoV-2 is a Beta coronavirus along with two profoundly pathogenic infections, SARS-CoV and MERS-CoV. SARS-CoV-2 is positive-sense single- stranded RNA (+ssRNA) infection [1]. In 2019 WHO named this virus as a coronavirus which was identified in throat and nasal swabs [2]. This pathogen belongs to Coronavirus Study Group hence this was renamed as severe acute respiratory syndrome coronavirus 2 (SARS-CoV-2) by WHO [3]. Later WHO officially named as coronavirus disease. In 2020, the WHO proclaimed that Coronavirus was a worldwide pandemic, demonstrating huge worldwide spread of an infectious disease [4]. This is a dreadful virus and contagious which leads to death and other severe complications. Patients with severe cases were admitted 
in intensive care units. Many vaccines were emerged to save the lives of people hence, vaccines can decrease the mortality rate and severe conditions. Human papillomavirus (HPV) In 1990 HPV vaccine was first developed by the University of Queensland. In recent analyses of the global burden cervical cancer in women is one of the major problems. The HPV16 (3.2\%) and HPV18 (1.4\%) are the prevalent types which are seen all around the world [5]. In developing countries more than $80 \%$ deaths occur in worldwide because in women's cervical carcinoma is the most familiar cancer [6]. HPV vaccine is shown to be productive in reducing cervical carcinoma with 16 and 18 HPV types $[7,8]$ Most of the cervical cancer cases are higher in developing countries (72 to $77 \%$ ) rather than less developed countries (65 to 72\%). HPV vaccination prevents $70 \%$ of cervical cancers which was reported in many RCTS [9]. WHO Introduced HPV vaccination in 100 countries to eliminate cervical cancer. A recent study in lancet found that $90 \%$ of Cervical cancer rates are almost lower in vaccinated women [10] In 2008 England, UK Introduced (HPV) bivalent immunization (Cervarix) which is a regular vaccination was given to girls at age of 12-13 years and 14-18 years. England initially used bivalent vaccine which gives protection against two HPV types A quadrivalent vaccine Gardasil has been used in September 2012. In England during the period of 2006-2019, for 7 cohorts of women aged 20 and 64. 28,000 cervical cancer cases were diagnosed and 300000 diagnosed for non-invasive cervical carcinoma (CIN3). By comparing three cohorts with non-vaccinated population, cervical cancers cases were just 638 and CIN3 cases were 18,662. According to International Agency for Research on Cancer HPV is one of the carcinogenic factors with different HPV types which are associated with $5 \%$ of all cancers.

\section{Materials and Methods}

Covid-19

The incidence has been higher in the pandemic given restricted admittance and prioritization of testing for selected groups. Since beginning of pandemic the incidence of covid-19 new cases is reported as 700000 . Every week the incidence of new cases as well as deaths were increased by $16 \%$ and $34 \%$. Hence mortality of Covid-19 per annum is 248,016 .

\section{Cervical cancer}

HPV vaccination can possibly decrease cervical malignant growth frequency all around the world by as much as $90 \%$. In the population the prevalence of HPV types can be reduced by Vac- cination $15 \%$ immunization coverage decreases the maximum prevalence of infection from $39 \%$ to $33 \%$, while $90 \%$ coverage diminishes the peak prevalence to $7 \%$ Mortality of Cervical cancer per annum is 60,078 .

\section{Burden of disease for Covid 19 and HPV}

- $\quad$ Total covid cases per million in the world 23,192.90

- In 2021 India has 12.5 million cases

- $\quad$ In India 397,668 deaths due to covid 19

- Globally 2.1 million cases of cervical cancer

- In India estimated New Cases in 2021 is 14,480

- In India 74000 deaths occur in every year.

\section{Results and Discussion}

Cost of Vaccine for COVID-19: Globally vaccines are more effective which can reduce high burden of diseases The development on Coronavirus immunizations were health care providers. Each year vaccines are saving millions of lives which are most cost-effective and reliable with better public health interventions. To stop spread of Corona virus and deaths uptake of vaccination is very much encouraged in the communities.

\begin{tabular}{|l|c|}
\hline Country & Cost of Vaccine \\
\hline India & Rs $700-1400$ \\
\hline United States & $\$ 2-\$ 16$ \\
\hline Europe & $12-15$ euros \\
\hline Russia & $\$ 6-\$ 10$ \\
\hline Brazil & $\$ 29-\$ 32$ \\
\hline South Africa & $\$ 3-\$ 5.25$ \\
\hline
\end{tabular}

Table 1: Cost of Covid-19 vaccines in Different countries.

\section{Cost of HPV vaccine for cervical cancer}

Cervical cancer can be prevented mostly by providing HPV vaccine to girls and women who were exposed to the virus there by HPV vaccination prevents vulvar, genital warts and vaginal cancers. As per Centres for Disease Control and Prevention (CDC) recommended HPV vaccination can be given to the 9-14 years girls. 
In United States three vaccines like Gardasil, Gardasil 9, and Cervarix have been licensed which can prevent infection by diseasecausing HPV. Since 2016 Gardasil 9 has been vaccinated for women in United States which can prevents the disease by different HPV types:

- $\quad 90 \%$ genital warts are caused by 6 and 11 HPV types [11]

- About $70 \%$ or even high percentage of cervical cancers are caused by high risk 16 and 18 HPV types [2]

- Mostly $10 \%$ to $20 \%$ HPV types like $31,45,33,58,52$ has chances of developing cervical cancer.

\begin{tabular}{|l|c|}
\hline Country & Cost of Vaccine \\
\hline India & Rs 410-760 [12] \\
\hline United States & $\$ 34$ \\
\hline Europe & $\$ 38$ \\
\hline
\end{tabular}

Table 2: Cost of HPV vaccines in Different countries.

Lives saved for Covid-19 vaccination

Total lives saved is $2,07,421$ which corresponds to $1,506,501$ QALYs gained. For limited effectiveness on transmission, 122,550 lives, which was equivalent 645,570 QALYs gained, were found to be saved [13].

Lives saved for HPV vaccination

- In the recent study it was estimated Lives saved by HPV vaccination for 9- to 14-year-old is lesser than 42, 000 annually [14].

- $\quad$ that the vaccinated cost for per girl was taken as US\$13.9 (INR 918), which encompasses with service delivery and cost of vaccine as (US\$ 8.8; INR 586). According to Indian strategy it was recommended ICER of (INR 54,881) per QALY gained [15].

Budgetary Impact for Covid-19: 35,000 crores allotted for COVID- 19 vaccination.

Covaxin- Rs 295 per dose

Covishield- Rs 200 per dose

Total 2 doses

Total population: 1.39 billion

- Vaccinating 1.39 billion people at Rs 590 (2 doses) will cost Rs $82,100 \mathrm{Cr}$.

- Vaccinating 1.39 billion people at Rs 400 (2 doses) will cost Rs 55,600 Cr.
But given circumstances we can vaccinate only $87.5 \mathrm{Cr}$ people (0.875 billion) at Rs 400 (2 doses) or $59.3 \mathrm{Cr}$ people (0.5932 billion) at Rs 590 (2 doses).

Range: Rs 55,600 Cr- Rs 82,100 Cr.

Target population: $59.3 \mathrm{Cr}-87.5 \mathrm{Cr}$ people.

\section{Budgetary Impact for HPV Vaccination}

Currently not included in the universal immunization scheme.

Price of the vaccine ranges from Rs 410- 760 with varying efficacy from $40 \%$ to $100 \%$. (Requires 1 to 3 doses depending on the age).

Population under study: 120 million

- Vaccinating 120 million girls at Rs 410 per dose will cost Rs 4920 Cr (2 doses- Rs 9840 Cr).

- Vaccinating 120 million girls at Rs 760 per dose will cost Rs 9120 Cr (2 doses- Rs 18240 Cr.).

Range: Rs 4920 Cr- Rs 9120 Cr (Rs 9840 Cr- Rs 18240 Cr.

\section{Cost effectiveness for Covid-19}

Nearly 160,000 patients' lives were saved and assumed 7.5 QALYs lost per death. During lockdowns ICER was found to be 28,500 USD/QALY $\left(=34,200,000,000 /\left(160,000 \_7.5\right)\right.$. Although, the estimation of vaccine cost effectiveness is higher than the real-life numbers [16]. 70\% of Vaccination to the population and its efficacy of two doses will result in 5132 (4926-5276) per QALY gained. As compared to vaccination verses no vaccination the US adult population has ICER per QALY gained the was $\$ 8,200$.

With less risk of severity and mortality in Hospitals the amount of QALY gained which is increased to $\$ 94,000$ [17].

Cost Effectiveness for HPV Vaccination: By implementing vaccination alone as compared to no vaccination the incremental cost per QALY gained was INR 5693. Likewise, when compared to no vaccination VIA 5 yearly and VIA 10 cohorts were screened it leads to (ICER per QALY gained) of US\$ 402 (INR 26,212) and US\$ 476 (INR 31,511) [18].

The COVID-19 infection was diagnosed in saliva or nasal swab where viral RNA can be detected. SARS-CoV-2's binds to its glycoproteins as vaccines produces mRNA cells that contains spikes for triggering antibody production so these covid-19 vaccines are use- 
ful for life saving and also reduces the cost of hospital stay and mortality rates can also be decreased. Although these vaccines are safe but in clinical trials participants had faced minor complications [19]. Women should go for screening at least twice in a life time, $90 \%$ HPV vaccination gives protection against the $16,18,31,33,45$, 52 , and $58 \mathrm{HPV}$ types. After screening and vaccination, the results states that cervical cancer can be eliminated [20]. As when compared with vaccinated women with Unvaccinated women $90 \%$ of Cervical cancer rates were decreased in women vaccinated against HPV. By providing vaccination for age 16-18 years cervical cancer rate was reduced to $34 \%, 12-13$-year school girls and 14-16 years reduced by $62 \%$ and $72-94$ years by $87 \%$.

\section{Conclusion}

In this article the two scenarios were explained the first scenario of Covid-19 finally concludes per Annum Mortality of Covid-19 is $(248,016)$ so, several vaccines for COVID 19 were emerged from different countries like India, United States, Europe, Russia, Brazil and South Africa form different companies. Total lives saved was $2,07,421$ which was corresponding to 1,506,501 QALYs gained. Government of India allocated 35000 crores for nation-wide vaccination programme. Vaccinating 1.39 billion people at Rs 590 (2 doses) will cost Rs 82,100 Cr. Vaccinating 1.39 billion people at Rs 400 ( 2 doses) will cost Rs $55,600 \mathrm{Cr}$. $70 \%$ of Vaccination to the population and its efficacy of two doses will result in 5132 (49265276) per QALY gained. As compared to vaccination verses no vaccination the US adult population has ICER per QALY gained the was $\$ 8,200$. As People from all over the world had taken Covid-19 mass vaccination which can reduce severity and mortality rates hence, a greater number of lives can be saved.

The second scenario of HPV vaccination finally concludes as Mortality of cervical cancer per annum is $(60,078)$ so, many countries along with India nationally introduce human papillomavirus (HPV) vaccine into their immunization programmes it primarily targets girls aged 9 to 14 years of age and total number of lives saved was lesser than 42000 annually. In the recent study it was estimated that the vaccinated cost for per girl was taken as US\$ 13.9 (INR 918), which encompasses with service delivery and cost of vaccine as (US\$ 8.8; INR 586). Vaccinating 120 million girls at Rs 410 per dose will cost Rs 4920 Cr (2 doses- Rs 9840 Cr). Vaccinating 120 million girls at Rs 760 per dose will cost Rs $9120 \mathrm{Cr}$ (2 doses- Rs 18240 Cr.). When compared to no vaccination VIA 5 yearly and VIA 10 cohorts were screened it leads to (ICER per QALY gained) of US\$ 402 (INR 26,212) and US\$ 476 (INR 31,511). women in India therefore, taking HPV vaccination will reduce cervical cancer burden in women and also mortality rates can be decreased. HPV vaccination can save number of lives of women. Recently National Comprehensive Cancer Network (NCCN) had recommended patients with cancer can take on COVID-19 vaccination.

\section{Acknowledgements}

I thank all those who have helped in the research work

\section{Conflict of Interest}

The authors declared that there is no conflict of interest.

\section{Bibliography}

1. Kramer A., et al. "How long do nosocomial pathogens persist on inanimate surfaces: A systematic review". BMC Infectious Disease 6 (2006): 130.

2. Hui D., et al. "The continuing 2019-nCoV epidemic threat of novel coronaviruses to global health: The latest 2019 novel coronavirus outbreak in Wuhan, China". International Journal of Infectious Diseases 91 (2020): 264-266.

3. Gorbalenya A., et al. "Severe acute respiratory syndrome-related coronavirus: the species and its viruses - a statement of the Coronavirus Study Group". BioRxiv (2020).

4. Rismanbaf A. "Potential Treatments for COVID-19 a Narrative Literature Review". Archives of Academic Emergency Medicine 8 (2020): e29.

5. David F., et al. "Global Burden of Human Papillomavirus and Related Diseases”. Vaccine 5 (2012): F12-F23.

6. Jan M., et al. "Introducing HPV Vaccine in Developing Countries: Key Challenges and Issues". The New England Journal of Medicine (2007): 356:19.

7. Barken S., et al. "Frequency of cervical intraepithelial neoplasia treatment in a well-screened population". International Journal of Cancer 130 (2012): 2438-2444.

8. Carter J., et al. "HPV infection and cervical disease: a review". Australian and New Zealand Journal of Obstetrics and Gynaecology 51 (2011): 103-108.

9. Clifford G., et al "HPV type-distribution in women with and without cervical neoplastic diseases". Vaccine 24. (2006). S26S34.

10. Munoz N., et al. "Epidemiologic classification of human papillomavirus types associated with cervical cancer". The New England Journal of Medicine 348. (2003): 518-527. 
11. De Martel C., et al. "Global burden of cancers attributable to infections in 2008: A review and synthetic analysis". Lancet Oncology 13 (2012): 607-615.

12. Shankar P., et al. "Cost-Effectiveness of Human Papillomavirus Vaccination for Adolescent Girls in Punjab State: Implications for India's Universal Immunization Program". Cancer 123.17 (2017): 3253-3260.

13. Koutsky L., et al. "A controlled trial of a human papillomavirus type 16 vaccine". New England Journal of Medicine 21 (2002): 1645-1651.

14. Arnold H., et al. "COVID-19 Vaccination Scenarios: A Cost-Effectiveness Analysis for Turkey". Vaccines (2021).

15. Mark J., et al. "Potential lives saved in 73 countries by adopting multi-cohort vaccination of 9-14-year-old girls against human papillomavirus". International Journal of Cancer 2. (2018): 317-323.

16. Akashdeep S., et al. "Cost effectiveness of strategies for cervical cancer prevention in India”. Plos One 15.9 (2020): e0238291.

17. Michael H., et al. "COVID-19 Vaccination Efficacy and Safety Literature Review". Journal of Clinical and Medical Research (2020).

18. Speiser D., et al. "COVID-19: Mechanisms of vaccination and immunity". Vaccines 3 (2020): 404.

19. Baden L., et al. "Efficacy and safety of the mRNA-1273 SARSCoV-2 vaccine". New England Journal of Medicine 5 (2021): 403-416.

20. Marc B., et al. "Impact of HPV vaccination and cervical screening on cervical cancer elimination: a comparative modelling analysis in 78 low-income and lower-middle-income countries". Lancet 395.10224 (2020): 575-590.

\section{Assets from publication with us}

- Prompt Acknowledgement after receiving the article

- Thorough Double blinded peer review

- Rapid Publication

- Issue of Publication Certificate

- High visibility of your Published work

Website: www.actascientific.com/

Submit Article: www.actascientific.com/submission.php

Email us: editor@actascientific.com

Contact us: +919182824667 